OPEN ACCESS

Edited by:

Giorgia Mori,

The University of Queensland,

Australia

Reviewed by:

Diana Machado,

New University of Lisbon, Portugal

Daniela Fernandes Ramos,

Federal University of Rio Grande,

Brazil

*Correspondence:

Jin-Town Wang

wangjt@ntu.edu.tw

Specialty section:

This article was submitted to

Antimicrobials, Resistance and

Chemotherapy,

a section of the journal

Frontiers in Microbiology

Received: 29 April 2020

Accepted: 24 June 2020

Published: 15 July 2020

Citation:

Hsu L-Y, Lai L-Y, Hsieh P-F, Lin T-L,

Lin $W-H$, Tasi $H-Y$, Lee $W-T$, Jou $R$ and Wang J-T (2020) Two Novel katG Mutations Conferring Isoniazid Resistance in

Mycobacterium tuberculosis.

Front. Microbiol. 11:1644.

doi: 10.3389/fmicb.2020.01644

\section{Two Novel katG Mutations Conferring Isoniazid Resistance in Mycobacterium tuberculosis}

\author{
Li-Yu Hsu', Li-Yin Lai', Pei-Fang Hsieh ${ }^{1}$, Tzu-Lung Lin', Wan-Hsuan Lin ${ }^{2,3}$, \\ Hsing-Yuan Tasi ${ }^{2,3}$, Wei-Ting Lee ${ }^{2,3}$, Ruwen Jou ${ }^{2,3}$ and Jin-Town Wang ${ }^{1,4 *}$ \\ ${ }^{1}$ Department of Microbiology, National Taiwan University College of Medicine, Taipei, Taiwan, ${ }^{2}$ Tuberculosis Research Center, \\ Taiwan Centers for Disease Control, Taipei, Taiwan, ${ }^{3}$ Diagnostics and Vaccine Center, Taiwan Centers for Disease Control, \\ Taipei, Taiwan, ${ }^{4}$ Department of Internal Medicine, National Taiwan University Hospital, Taipei, Taiwan
}

Tuberculosis (TB), an infectious disease caused by Mycobacterium tuberculosis, is among the top 10 leading causes of death worldwide. The treatment course for TB is challenging; it requires antibiotic administration for at least 6 months, and bacterial drug resistance makes treatment even more difficult. Understanding the mechanisms of resistance is important for improving treatment. To investigate new mechanisms of isoniazid $(\mathrm{INH})$ resistance, we obtained three $\mathrm{INH}$-resistant $(\mathrm{INH}-\mathrm{R}) \mathrm{M}$. tuberculosis clinical isolates collected by the Taiwan Centers for Disease Control (TCDC) and sequenced genes known to harbor $\mathrm{INH}$ resistance-conferring mutations. Then, the relationship between the mutations and INH resistance of these three $I N H-R$ isolates was investigated. Sequencing of the INH-R isolates identified three novel katG mutations resulting in R146P, W341R, and L398P KatG proteins, respectively. To investigate the correlation between the observed $\mathrm{INH}-\mathrm{R}$ phenotypes of the clinical isolates and these $\mathrm{kat} G$ mutations, wild-type kat $G$ from $\mathrm{H} 37 \mathrm{R} v$ was expressed on a plasmid (pMN437-katG) in the isolates, and their susceptibilities to INH were determined. The plasmid expressing H37Rv katG restored INH susceptibility in the two INH-R isolates encoding the W341R KatG and L398P KatG proteins. In contrast, no phenotypic change was observed in the KatG R146P isolate harboring pMN437-katG. H37Rv isogenic mutant with W341R KatG or L398P KatG was further generated. Both showed resistant to INH. In conclusion, W341R KatG and L398P KatG conferred resistance to INH in M. tuberculosis, whereas R146P KatG did not affect the INH susceptibility of M. tuberculosis.

Keywords: Mycobacterium tuberculosis, drug resistance, isoniazid, mutation, katG

\section{INTRODUCTION}

According to the Global Tuberculosis Report published by the World Health Organization (WHO), tuberculosis (TB), the airborne infectious disease caused by Mycobacterium tuberculosis (Cambau and Drancourt, 2014), is one of the top 10 causes of death worldwide, and thus remains a major global public health problem (WHO, 2019). The emergence of drug-resistant TB has made the need for improvements in diagnostic accuracy and successful treatment even more urgent, as both are major challenges in TB control and key causes of its high mortality rate (Nguyen et al., 2019). 
Since the 1940s, several drugs have been developed for the treatment of TB (Sotgiu et al., 2015; Kurz et al., 2016). These drugs can be classified as first-line anti-TB drugs, including the isoniazid (INH), rifampicin, pyrazinamide, and ethambutol, as well as other second-line drugs, which are used in cases of treatment failure (Rendon et al., 2016). The first-line anti-TB drug, INH, which was initially shown to have anti-TB activity in 1952 (Fox, 1952), is suitable for treatment when M. tuberculosis is replicating (Chakraborty and Rhee, 2015). INH is a prodrug that is activated by the catalase-peroxidase KatG. The metabolites produced then react with nicotinamide adenine dinucleotide $\left(\mathrm{NAD}^{+}\right)$, and binding of the INH-NAD adduct to the $\mathrm{NADH}$-dependent enoyl-ACP reductase InhA. InhA inhibits mycolic acid formation and cell wall synthesis in M. tuberculosis, leading to cell death (Vilchèze and Jacobs, 2014; Chakraborty and Rhee, 2015; Islam et al., 2017).

Most INH-resistant (INH-R) strains harbor mutations in genes associated with cell wall synthesis, the kat $G$ gene (Somoskovi et al., 2001; Laurenzo and Mousa, 2011), the inhA gene and its promoter (Zhang et al., 1992; Nguyen et al., 2019), or the oxyR-ahpC region (Sreevatsan et al., 1997; Lempens et al., 2018). Articles suggest that $k a t G$ deletion mutants have higher INH resistance than strains with mutations in inhA or its promoter (Somoskovi et al., 2001; Lempens et al., 2018). In addition, upregulation of INH inactivators or efflux pumps was involved in INH resistance (Vilchèze and Jacobs, 2014; Unissa et al., 2016).

In this work, three INH-R clinical isolates with minimum inhibitory concentrations (MICs) $\geq 64 \mathrm{mg} / \mathrm{L}$ were found to have novel kat $G$ mutations that were not previously reported to confer INH resistance. The aim of this study was to examine whether the amino acid changes encoded by the kat $G$ mutations in these high-level INH-R clinical isolates are determinants of INH resistance. We compared the INH susceptibility of these isolates to those of isolates expressing the H37Rv KatG protein, and then recreated these point mutations in H37Rv to confirm the relationship between the kat $G$ mutations and INH resistance.

\section{MATERIALS AND METHODS}

\section{Ethics Statement}

According to the Taiwan Communicable Disease Control Act, $\mathrm{TB}$ is one of the notifiable diseases, and specimen collection for laboratory testing is mandatory. This study did not require ethics approval, and participant consent was not required.

\section{Bacterial Strains and Culture Conditions}

The bacterial strains used in this study are listed in Supplementary Table S1. All the experiments for M. tuberculosis strains were carried out at a BSL-3 laboratory in National Taiwan University College of Medicine, Taiwan, following

Nomenclature: TB, Tuberculosis; INH, Isoniazid; INH-R, INH-resistant; WHO, World Health Organization; $\mathrm{NAD}^{+}$, Nicotinamide adenine dinucleotide; MICs, Minimum inhibitory concentrations; TCDC, Taiwan Centers for Disease Control; OADC, Oleic acid/albumin/dextrose/catalase; LB, Luria-Bertani; PCR, Polymerase chain reaction. institutional biosafety procedures. The INH-R M. tuberculosis isolates identified by clinical TB laboratories of Taiwan were sent to the Reference laboratory of Mycobacteriology at the Taiwan Centers for Disease Control (TCDC) for confirmation. Reference strain M. tuberculosis $\mathrm{H} 37 \mathrm{Rv}, \mathrm{H} 37 \mathrm{Rv}$-derived isogenic mutants, and the clinical INH-R isolates were grown in Middlebrook 7H9 liquid medium (BD Difco, Sparks, MD, USA) containing $10 \%$ oleic acid/albumin/dextrose/catalase (OADC; BD Difco, Sparks, MD, USA), 0.5\% glycerol, and 0.05\% Tween-80 or Middlebrook 7H11 solid agar (BD Difco, Sparks, MD, USA) containing $10 \%$ OADC and $0.5 \%$ glycerol at $37^{\circ} \mathrm{C}$. Escherichia coli $\mathrm{DH} 10 \mathrm{~B}$, for plasmid construction, was grown in Luria-Bertani (LB) medium (Bio Basic, Toronto, Canada) at $37^{\circ} \mathrm{C}$. The following were added to medium as needed for selection: $50 \mathrm{mg} / \mathrm{L}$ hygromycin (BioShop, Ontario, Canada), $100 \mathrm{mg} / \mathrm{L} \mathrm{X-gal,} \mathrm{and}$ $4 \%$ sucrose for $M$. tuberculosis and $100 \mathrm{mg} / \mathrm{L}$ hygromycin (BioShop, Ontario, Canada) for E. coli.

\section{Screening and Sequencing of INH-R Clinical Isolates}

The MIC of INH-R M. tuberculosis isolates was screened and determined using the Sensititre ${ }^{\mathrm{TM}}$ MYCOTB MIC Plate (Trek Diagnostic Systems, OH, USA) according to the manufacturer's instructions. The ranges of drug concentrations were $0.03-4 \mathrm{mg} / \mathrm{L}$ for INH. The bacterial solution was adjusted to turbidity at a McFarland standard of 0.5 and then added to the Sensititre ${ }^{\mathrm{TM}}$ MYCOTB MIC Plate before the plate was covered with the adhesive plastic seal. After incubation at $37^{\circ} \mathrm{C}$ for 14 or 21 days, the results were recorded using the Sensititre ${ }^{\mathrm{TM}}$ Vizion $^{\mathrm{TM}}$ Digital MIC Viewing System.

The primers used to sequences $k a t G$, inhA, oxyR, and $a p h C$ are listed in Supplementary Table S2. The polymerase chain reaction (PCR) cycling conditions were previously described (Jou et al., 2019). The sequences of $k a t G$ in INH-R strains CDC-A, CDC-B, and CDC-C had been submitted to NCBI (GenBank accession numbers: MT572851, MT572852, and MT572853).

\section{KatG Expression Constructs}

The primer and plasmids used in this study are listed in Supplementary Tables S2, S3. To express wild-type kat $G$ in the INH-R clinical strains, a $k a t G$ expression plasmid, pMN437-kat $G$, was generated by ligating the kat $G$ gene from $M$. tuberculosis H37Rv to pMN437 (Song et al., 2008; Steinhauer et al., 2010), which was linearized via reverse PCR to remove the $g f p$ gene. The pMN437 and pMN437-kat $G$ plasmids were transformed into competent $M$. tuberculosis cells by electroporation at $2,500 \mathrm{~V}$, $1,000 \Omega$, and $25 \mu \mathrm{F}$ as previously described (Larsen et al., 2007).

\section{Mutagenesis of katG in H37Rv}

To replace the kat $G$ gene in $\mathrm{H} 37 \mathrm{Rv}$ with the mutant genes in the INH-R clinical isolates, fragments of the mutated kat $G$ genes in clinical strains CDC-A and CDC-B were amplified and ligated into the pGOAL19 plasmid at the ScaI site. The primers used are listed in Supplementary Table S2. The pGOAL19 plasmid is a suicide plasmid that lacks a mycobacterial origin for plasmid replication (Parish and Stoker, 2000). 
When the pGOAL19 recombinant plasmid (pGOAL19-katG W341R R463L or pGOAL19-katG L398P R463L) was transformed to the $\mathrm{H} 37 \mathrm{Rv}$ strain, a two-step homologous recombination has occurred between the plasmid and the genome results in replacement of the $\mathrm{H} 37 \mathrm{Rv}$ gene with the mutant gene. The $\mathrm{H} 37 \mathrm{Rv}$ isogenic mutants were confirmed by PCR and sequencing.

\section{INH Susceptibility Tests}

To evaluate the effect of $\mathrm{H} 37 \mathrm{Rv}$ KatG expression in INH-R isolates and the impact of KatG from the INH-R isolates in $\mathrm{H} 37 \mathrm{Rv}$ isogenic mutants, the INH susceptibility was assessed by the agar dilution assay. Briefly, $5 \mu \mathrm{l}$ of a $4 \times 10^{6} \mathrm{CFU} / \mathrm{ml}$ bacterial suspension (equivalent to $2 \times 10^{4} \mathrm{cfu}$ ) was spotted on Middlebrook 7H11 agar plates containing 10\% OADC, 0.5\% glycerol, and serial diluted INH (Sigma, St. Louis, MO, USA) concentrations of $0,0.2,1,4,16$, and $64 \mathrm{mg} / \mathrm{L}$ and incubated at $37^{\circ} \mathrm{C}$. The results were recorded after 3 weeks of incubation. Resistance was defined as colonies growing in the presence of the critical concentrations of $0.2 \mathrm{mg} / \mathrm{L} \mathrm{INH}$, according to the CLSI guidelines (Clinical and Laboratory Standards Institute, 2011). All reported MICs were represented from three independent experiments. For the H37Rv KatG expression, H37Rv/pMN437 was used as the control strain, and $50 \mathrm{mg} / \mathrm{L}$ hygromycin was added to the agar plates to maintain the transformed plasmids. To compare the INH susceptibility of KatG from the clinical isolates in $\mathrm{H} 37 \mathrm{Rv}$ isogenic mutants, $\mathrm{H} 37 \mathrm{Rv}$ was used as the control.

\section{RESULTS}

\section{Three INH-R Clinical Isolates Harbor Novel Mutations in katG}

Three INH-R strains, CDC-A, CDC-B, and CDC-C, were provided by TCDC. The MICs of the three INH-R isolates were all $>4 \mathrm{mg} / \mathrm{L}$, which were initially determined using the Sensititre $^{\mathrm{TM}}$ MYCOTB MIC Plate. To identify the uncommon mutations that conferred INH resistance in these isolates, we sequenced the frequent INH resistance hotspots of kat $G$, $\operatorname{inh} A$, and $\operatorname{oxy} R$-aphC regions. The results demonstrated that the three isolates were carried wild-type inhA, oxyR, and $a p h C$ genes and had mutations in the kat $G$ gene resulting in the following amino acid changes: W341R (TGG/CGG), L398P (CTG/CCG), and R146P (CGG/CCG), respectively. All of them also harbored the R463L (CGG/CTG) change in KatG, and this residue change has been confirmed earlier as a polymorphism (Figure 1). Then, we further confirm the INH susceptibilities of all three isolates and revealed those were $>64 \mathrm{mg} / \mathrm{L}$ by the agar dilution assay (Figure 2). The results indicate that the three amino acid residues change in kat $G$ might result in $\mathrm{INH}$ resistance in our INH-R isolates.

\section{H37Rv KatG-Complemented Clinical Strains Carrying W341R and L398P KatG Are More Susceptible to INH}

To delineate the association of the W341R, L398P, and R146P KatG proteins with the INH resistance in the isolates CDC-A,

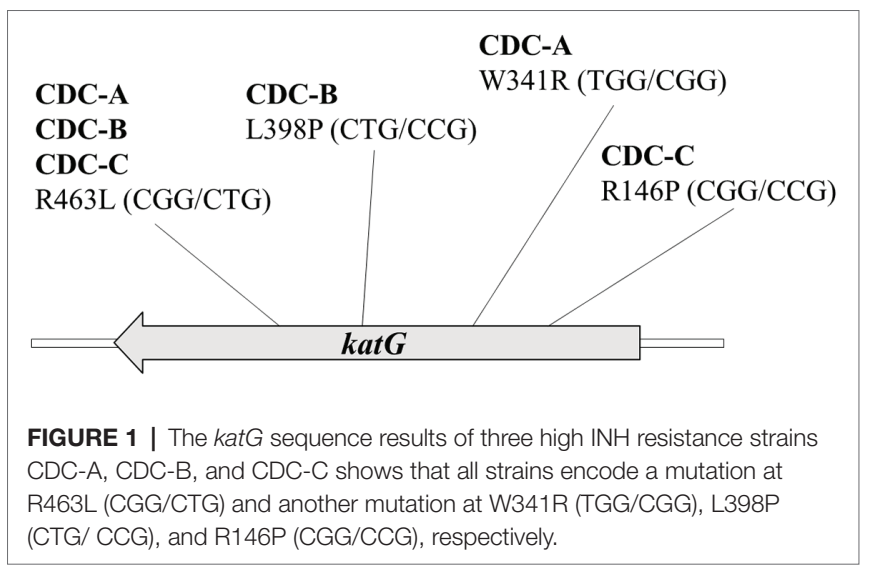

CDC-B, and CDC-C, we expressed the KatG protein from H37Rv in these INH-R strains using pMN437-katG. To examine whether expression of $\mathrm{H} 37 \mathrm{Rv}$ KatG could restore INH resistance, INH susceptibility tests were conducted (Figure 3). The results showed that CDC-A/pMN437-kat $G$ was more susceptible to INH $(\mathrm{MIC}<4 \mathrm{mg} / \mathrm{L})$ than the CDC-A carrying an empty vector (CDC-A/pMN437; MIC >64 mg/L). CDC-B/pMN437-katG was INH sensitive $(\mathrm{MIC}<0.2 \mathrm{mg} / \mathrm{L})$, whereas $\mathrm{CDC}-\mathrm{B} / \mathrm{pMN} 437$ was highly resistant (MIC $>64 \mathrm{mg} / \mathrm{L}$ ). In contrast, CDC-C/ pMN437-katG showed no difference in INH susceptibility when compared with CDC-C/pMN437 (Table 1). Therefore, we concluded that KatG W341R and L398P are the resistanceconferring amino acid changes in strains CDC-A and CDC-B, respectively. In contrast, KatG $\mathrm{R} 146 \mathrm{P}$ is not the determinant for $\mathrm{INH}$ resistance of the CDC-C strain.

\section{The W341R and L398P KatG Proteins Confer INH Resistance in M. tuberculosis}

To directly confirm the contribution of the W341R and L398P KatG proteins on INH resistance, the katG gene of $\mathrm{H} 37 \mathrm{Rv}$ was replaced with a segment of the DNA from the clinical isolates containing these kat $G$ mutations. The DNA sequencing of the H37Rv isogenic mutants H37Rv katG W341R and H37Rv $k a t G$ L398P R463L showed that the katG of $\mathrm{H} 37 \mathrm{Rv}$ was successfully substituted with the kat $G$ gene from CDC-A and CDC-B, respectively. The INH susceptibilities of the H37Rv isogenic mutants $\mathrm{H} 37 \mathrm{Rv}$ katG W341R and H37Rv katG L398P R463L were measured by the agar dilution method (Figure 2), and revealed that, like $\mathrm{CDC}-\mathrm{A}$ and $\mathrm{CDC}-\mathrm{B}$, both strains were resistant to the highest INH concentration tested $(64 \mathrm{mg} / \mathrm{L})$, indicating a $\mathrm{MIC}>64 \mathrm{mg} / \mathrm{L}$ and demonstrating that $k a t G \mathrm{~W} 341 \mathrm{R}$ and L398P confer INH resistance (Table 2).

\section{DISCUSSIONS}

INH is a first-line TB drug, and researchers have clarified various mechanisms of INH resistance by collecting INH-R strains and identifying the mutated genes. Studies have shown that $50-94 \%$ INH-R strains have at least one mutation in $k a t G$, $10-35 \%$ INH-R strains carry at least one mutation in the inhA 


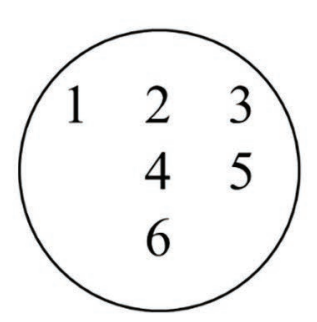

INH

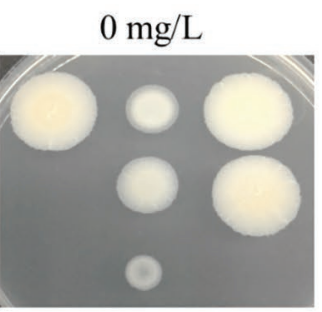

1: $\mathrm{H} 37 \mathrm{Rv}$

2: CDC-A

3: H37Rv kat G W341R

4: CDC-B

5: H37Rv katG L398P R463L

6: CDC-C
INH

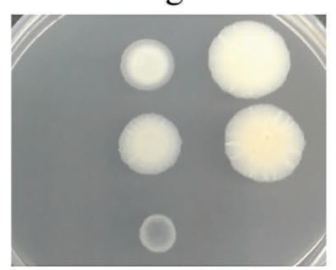

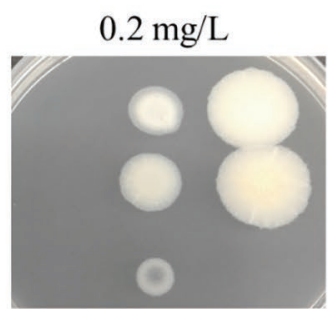

$16 \mathrm{mg} / \mathrm{L}$

$1 \mathrm{mg} / \mathrm{L}$

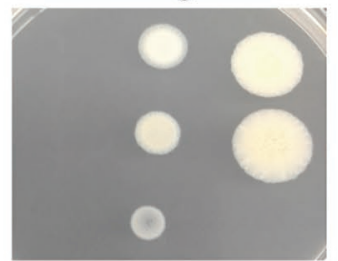

$64 \mathrm{mg} / \mathrm{L}$
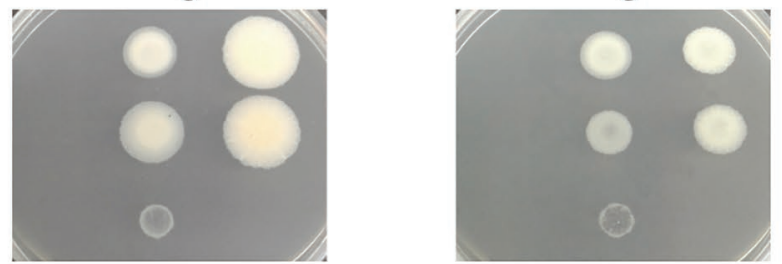

FIGURE 2 | INH susceptibility testing of the control (H37Rv, spot 1), clinical isolates [CDC-A (spot 2), CDC-B (spot 4), and CDC-C (spot 6)] and H37Rv isogenic mutants [H37Rv katG W341R (spot 3), and H37Rv katG L398P R463L (spot 5)].

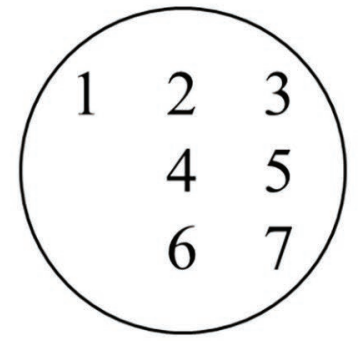

1: H37Rv/pMN437

2: CDC-A/pMN437

3: CDC-A/pMN437-katG

4: $\mathrm{CDC}-\mathrm{B} / \mathrm{pMN} 437$

5: CDC-B/pMN437-katG

6: $\mathrm{CDC}-\mathrm{C} / \mathrm{pMN} 437$

7: CDC-C/pMN437-katG
INH

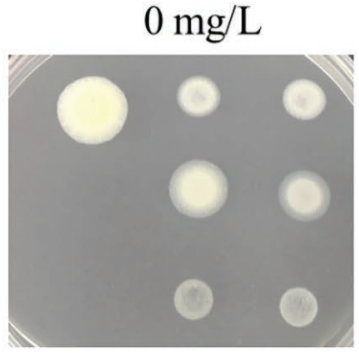

$4 \mathrm{mg} / \mathrm{L}$

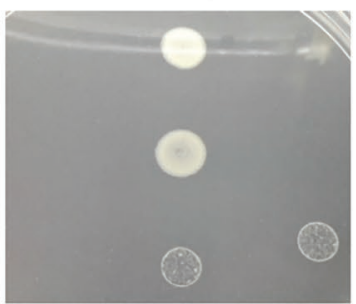

$0.2 \mathrm{mg} / \mathrm{L}$

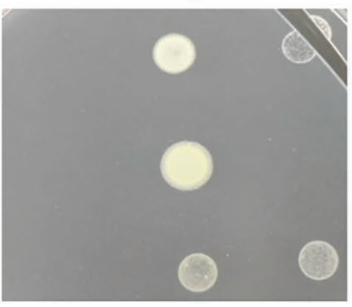

$16 \mathrm{mg} / \mathrm{L}$

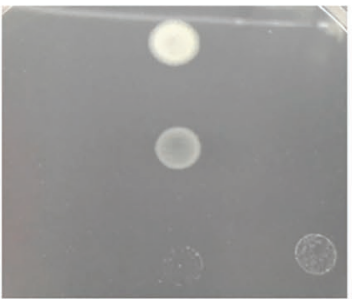

$1 \mathrm{mg} / \mathrm{L}$

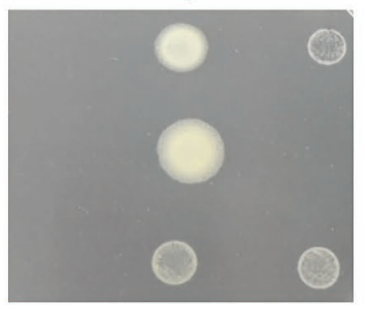

$64 \mathrm{mg} / \mathrm{L}$

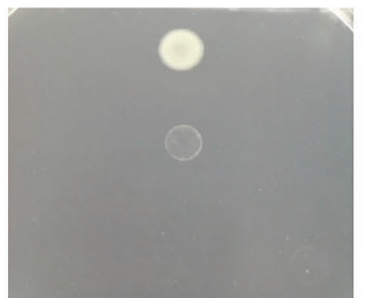

FIGURE 3 | Complementation of INH resistance in the clinical isolates. INH susceptibility testing of the control H37Rv carrying the empty vector pMN437 (H37Rv/ pMN437, spot 1); clinical isolate control strains carrying the empty vector pMN437 [CDC-A/pMN437 (spot 2), CDC-B/pMN437 (spot 4), and CDC-C/pMN437 (spot 6)]; and clinical isolates carrying wild-type katG expression plasmid [CDC-A/pMN437-katG (spot 3), CDC-B/pMN437-katG (spot 5), and CDC-C/pMN437-katG (spot 7)].

promoter, and $10-40 \%$ INH-R strains carry at least one mutation in $\operatorname{xxyR}$-ahpC region ( $\mathrm{Li}$ et al., 2015). The katG 315 mutation, which leads to high-level INH resistance and the inhA-15 mutation, which leads to low-level INH resistance, are two of the most common mutations (Yao et al., 2010; Zenteno-Cuevas et al., 2019). However, mutations in the oxyR-ahpC region have not yet been recorded to confer INH resistance directly (Kandler et al., 2018). Studies reported that the expression level of alkylhydroperoxidase (AhpC) was different in INH-R strains with mutations in $k a t G$, indicating that $A h p C$ were compensated for the loss of KatG activity and restored the anti-oxidative stress capacity (Ng et al., 2004; Nieto et al., 2016).
In this study, the KatG sequences of isolates CDC-A, CDC-B, and CDC-C revealed that each had a mutation at R463L (CGG/ CTG) and had another mutation, which have not been previously reported as INH resistance-conferring mutations at W341R (TGG/ CGG), L398P (CTG/CCG), and R146P (CGG/CCG), respectively. KatG R463L, was previously identified as a polymorphism, which is irrelevant to INH resistance (Johnsson et al., 1997; Ramaswamy et al., 2003; Lempens et al., 2018), and an epidemiological marker for Beijing strains (Tsolaki et al., 2005).

There are three studies, which had mentioned the mutations at KatG W341 in INH-R M. tuberculosis. A genetic analysis by next-generation sequencing performed in Ukraine. 
TABLE 1 | INH sensitivity test results for Mycobacterium tuberculosis clinical isolates with and without H37Rv KatG expression plasmid.

\begin{tabular}{lcl}
\hline Strains & INH MIC (mg/L) & Susceptibility \\
\hline H37Rv/pMN437 & $<0.2$ & Sensitive \\
CDC-A/pMN437 & $>64$ & Resistant \\
CDC-A/pMN437-katG & $\leq 4(>1)$ & Resistant \\
CDC-B/pMN437 & $>64$ & Resistant \\
CDC-B/pMN437-katG & $<0.2$ & Sensitive \\
CDC-C/pMN437 & $>64$ & Resistant \\
CDC-C/pMN437-katG & $>64$ & Resistant \\
\hline
\end{tabular}

INH, isoniazid; MIC, minimum inhibitory concentration.

TABLE 2 | Drug susceptibility testing of clinical isolates and H37Rv isogenic mutants harboring mutant katG.

\begin{tabular}{lcl}
\hline Strains & INH MIC (mg/L) & Susceptibility \\
\hline H37Rv & $<0.2$ & Sensitive \\
H37Rv katG W341R & $>64$ & Resistant \\
H37Rv katG L398P R463L & $>64$ & Resistant \\
CDC-A & $>64$ & Resistant \\
CDC-B & $>64$ & Resistant \\
\hline
\end{tabular}

INH, isoniazid; MIC, minimum inhibitory concentration.

Daum et al. found a poly-resistant strain of $M$. tuberculosis containing the amino acid substitutions W341R and R463L in KatG (Daum et al., 2018), which are the same amino acid changes as strain CDC-A. A strain with MIC $>1 \mathrm{mg} / \mathrm{L}$ found to have mutations at W341G and R463L by DNA sequencing (Brossier et al., 2006); and a group in Brazil found a strain with an INH MIC $>32 \mathrm{mg} / \mathrm{L}$ harbors KatG W341S (Cardoso et al., 2004). In this study, the MIC of INH for H37Rv katG W341R confirmed that W341R is the INH resistance determinant in M. tuberculosis. Therefore, we concluded that amino acid residue 341 of KatG could be crucial for INH resistance in $M$. tuberculosis.

The INH resistance of strain CDC-C could not be reversed by expression of H37Rv KatG. Therefore, we concluded that the mechanism underlying INH resistance in the CDC-C strain may involve another INH-associated gene than those studied or other non-explored resistance mechanism.

The mutagenesis system described here could be adapted in future studies of drug resistance mechanisms in M. tuberculosis

\section{REFERENCES}

Brossier, F., Veziris, N., Truffot-Pernot, C., Jarlier, V., and Sougakoff, W. (2006). Performance of the genotype MTBDR line probe assay for detection of resistance to rifampin and isoniazid in strains of Mycobacterium tuberculosis with low- and high-level resistance. J. Clin. Microbiol. 44, 3659-3664. doi: 10.1128/JCM.01054-06

Cambau, E., and Drancourt, M. (2014). Steps towards the discovery of Mycobacterium tuberculosis by Robert Koch, 1882. Clin. Microbiol. Infect. 20, 196-201. doi: 10.1111/1469-0691.12555

Cardoso, R. F., Cooksey, R. C., Morlock, G. P., Barco, P., Cecon, L., Forestiero, F., et al. (2004). Screening and characterization of mutations in isoniazidresistant Mycobacterium tuberculosis isolates obtained in Brazil. Antimicrob. Agents Chemother. 48, 3373-3381. doi: 10.1128/AAC.48.9.33733381.2004 to establish a more reliable genetic diagnosis. Once the resistanceconferring mutation is identified, clinical institutions could shorten the time for drug susceptibility testing to facilitate the control and treatment of drug-resistant M. tuberculosis.

\section{DATA AVAILABILITY STATEMENT}

The original contributions presented in the study are included in the article/Supplementary Material, and further inquiries can be directed to the corresponding author.

\section{AUTHOR CONTRIBUTIONS}

J-TW performed experiment conception and design. W-HL, H-YT, W-TL, and RJ performed clinical strains analysis. L-YH and L-YL performed investigation. L-YH, L-YL, T-LL, and $\mathrm{P}-\mathrm{FH}$ performed data analysis. L-YH, P-FH, and J-TW wrote manuscript. All authors contributed to the article and approved the submitted version.

\section{FUNDING}

This work was supported by grants from the Ministry of Science and Technology, the Excellent Translational Medicine Research Projects of National Taiwan University College of Medicine and National Taiwan University Hospital, the Liver Disease Prevention and Treatment Research Foundation of Taiwan, Taiwan Centers for Disease Control, and the "Center of Precision Medicine" from The Featured Areas Research Center Program within the framework of the Higher Education Sprout Project by the Ministry of Education (MOE).

\section{SUPPLEMENTARY MATERIAL}

The Supplementary Material for this article can be found online at: https://www.frontiersin.org/articles/10.3389/fmicb.2020.01644/ full\#supplementary-material. 
Johnsson, K., Froland, W. A., and Schultz, P. G. (1997). Overexpression, purification, and characterization of the catalase-peroxidase KatG from Mycobacterium tuberculosis. J. Biol. Chem. 272, 2834-2840. doi: 10.1074/ jbc.272.5.2834

Jou, R., Lee, W. T., Kulagina, E. V., Weng, J. Y., Isakova, A. I., Lin, W. H., et al. (2019). Redefining MDR-TB: comparison of Mycobacterium tuberculosis clinical isolates from Russia and Taiwan. Infect. Genet. Evol. 72, 141-146. doi: 10.1016/j.meegid.2018.12.031

Kandler, J. L., Mercante, A. D., Dalton, T. L., Ezewudo, M. N., Cowan, L. S., Burns, S. P., et al. (2018). Validation of novel Mycobacterium tuberculosis isoniazid resistance mutations not detectable by common molecular tests. Antimicrob. Agents Chemother. 62, e00974-e01018. doi: 10.1128/ AAC.00974-18

Kurz, S. G., Furin, J. J., and Bark, C. M. (2016). Drug-resistant tuberculosis: challenges and progress. Infect. Dis. Clin. North Am. 30, 509-522. doi: 10.1016/j.idc.2016.02.010

Larsen, M. H., Biermann, K., Tandberg, S., Hsu, T., and Jacobs, W. R. Jr. (2007). Genetic manipulation of Mycobacterium tuberculosis. Curr. Protoc. Microbiol. Chapter 10, Unit 10A.2. doi: 10.1002/9780471729259.mc10a02s6

Laurenzo, D., and Mousa, S. A. (2011). Mechanisms of drug resistance in Mycobacterium tuberculosis and current status of rapid molecular diagnostic testing. Acta Trop. 119, 5-10. doi: 10.1016/j.actatropica.2011.04.008

Lempens, P., Meehan, C. J., Vandelannoote, K., Fissette, K., De Rijk, P., Van Deun, A., et al. (2018). Isoniazid resistance levels of Mycobacterium tuberculosis can largely be predicted by high-confidence resistance-conferring mutations. Sci. Rep. 8:3246. doi: 10.1038/s41598-018-21378-x

Li, G., Zhang, J., Guo, Q., Jiang, Y., Wei, J., Zhao, L. -L., et al. (2015). Efflux pump gene expression in multidrug-resistant Mycobacterium tuberculosis clinical isolates. PLoS One 10:e0119013. doi: 10.1371/journal.pone.0119013

Ng, V. H., Cox, J. S., Sousa, A. O., Macmicking, J. D., and Mckinney, J. D. (2004). Role of KatG catalase-peroxidase in mycobacterial pathogenesis: countering the phagocyte oxidative burst. Mol. Microbiol. 52, 1291-1302. doi: 10.1111/j.1365-2958.2004.04078.x

Nguyen, T. N. A., Anton-Le Berre, V., Bañuls, A. -L., and Nguyen, T. V. A. (2019). Molecular diagnosis of drug-resistant tuberculosis; a literature review. Front. Microbiol. 10:794. doi: 10.3389/fmicb.2019.00794

Nieto, R. L. M., Mehaffy, C., Creissen, E., Troudt, J., Troy, A., Bielefeldt-Ohmann, H., et al. (2016). Virulence of Mycobacterium tuberculosis after acquisition of isoniazid resistance: individual nature of katG mutants and the possible role of AhpC. PLoS One 11:e0166807. doi: 10.1371/journal.pone.0166807

Parish, T., and Stoker, N. G. (2000). Use of a flexible cassette method to generate a double unmarked Mycobacterium tuberculosis tlyA plcABC mutant by gene replacement. Microbiology 146, 1969-1975. doi: 10.1099/ 00221287-146-8-1969

Ramaswamy, S. V., Reich, R., Dou, S. J., Jasperse, L., Pan, X., Wanger, A., et al. (2003). Single nucleotide polymorphisms in genes associated with isoniazid resistance in Mycobacterium tuberculosis. Antimicrob. Agents Chemother. 47, 1241-1250. doi: 10.1128/AAC.47.4.1241-1250.2003

Rendon, A., Tiberi, S., Scardigli, A., D’ambrosio, L., Centis, R., Caminero, J. A., et al. (2016). Classification of drugs to treat multidrug-resistant tuberculosis (MDR-TB): evidence and perspectives. J. Thorac. Dis. 8, 2666-2671. doi: $10.21037 /$ itd.2016.10.14
Somoskovi, A., Parsons, L. M., and Salfinger, M. (2001). The molecular basis of resistance to isoniazid, rifampin, and pyrazinamide in Mycobacterium tuberculosis. Respir. Res. 2, 164-168. doi: 10.1186/rr54

Song, H., Sandie, R., Wang, Y., Andrade-Navarro, M. A., and Niederweis, M. (2008). Identification of outer membrane proteins of Mycobacterium tuberculosis. Tuberculosis 88, 526-544. doi: 10.1016/j.tube.2008.02.004

Sotgiu, G., Centis, R., D’ambrosio, L., and Migliori, G. B. (2015). Tuberculosis treatment and drug regimens. Cold Spring Harb. Perspect. Med. 5:a017822. doi: $10.1101 /$ cshperspect.a017822

Sreevatsan, S., Pan, X., Zhang, Y., Deretic, V., and Musser, J. M. (1997). Analysis of the oxyR-ahpC region in isoniazid-resistant and -susceptible Mycobacterium tuberculosis complex organisms recovered from diseased humans and animals in diverse localities. Antimicrob. Agents Chemother. 41, 600-606. doi: 10.1128/AAC.41.3.600

Steinhauer, K., Eschenbacher, I., Radischat, N., Detsch, C., Niederweis, M., and Goroncy-Bermes, P. (2010). Rapid evaluation of the mycobactericidal efficacy of disinfectants in the quantitative carrier test EN 14563 by using fluorescent Mycobacterium terrae. Appl. Environ. Microbiol. 76, 546-554. doi: 10.1128/AEM.01660-09

Tsolaki, A. G., Gagneux, S., Pym, A. S., Goguet De La Salmoniere, Y. -O. L., Kreiswirth, B. N., Van Soolingen, D., et al. (2005). Genomic deletions classify the Beijing/W strains as a distinct genetic lineage of Mycobacterium tuberculosis. J. Clin. Microbiol. 43, 3185-3191. doi: 10.1128/JCM.43.7.3185-3191.2005

Unissa, A. N., Subbian, S., Hanna, L. E., and Selvakumar, N. (2016). Overview on mechanisms of isoniazid action and resistance in Mycobacterium tuberculosis. Infect. Genet. Evol. 45, 474-492. doi: 10.1016/j.meegid.2016.09.004

Vilchèze, C., and Jacobs, W. R. Jr. (2014). Resistance to isoniazid and ethionamide in Mycobacterium tuberculosis: genes, mutations, and causalities. Microbiol. Spectr. 2. doi: 10.1128/microbiolspec.MGM2-0014-2013

WHO (2019). Global tuberculosis report 2019. World Health Organization.

Yao, C., Zhu, T., Li, Y., Zhang, L., Zhang, B., Huang, J., et al. (2010). Detection of rpoB, katG and inhA gene mutations in Mycobacterium tuberculosis clinical isolates from Chongqing as determined by microarray. Clin. Microbiol. Infect. 16, 1639-1643. doi: 10.1111/j.1469-0691.2010.03267.x

Zenteno-Cuevas, R., Cuevas-Cordoba, B., and Parissi-Crivelli, A. (2019). rpoB, katG and inhA mutations in multi-drug resistant strains of Mycobacterium tuberculosis clinical isolates from Southeast Mexico. Enferm. Infecc. Microbiol. Clin. 37, 307-313. doi: 10.1016/j.eimc.2018.09.002

Zhang, Y., Heym, B., Allen, B., Young, D., and Cole, S. (1992). The catalaseperoxidase gene and isoniazid resistance of Mycobacterium tuberculosis. Nature 358, 591-593. doi: $10.1038 / 358591 \mathrm{a} 0$

Conflict of Interest: The authors declare that the research was conducted in the absence of any commercial or financial relationships that could be construed as a potential conflict of interest.

Copyright (๑) $2020 \mathrm{Hsu}$, Lai, Hsieh, Lin, Lin, Tasi, Lee, Jou and Wang. This is an open-access article distributed under the terms of the Creative Commons Attribution License (CC BY). The use, distribution or reproduction in other forums is permitted, provided the original author(s) and the copyright owner(s) are credited and that the original publication in this journal is cited, in accordance with accepted academic practice. No use, distribution or reproduction is permitted which does not comply with these terms. 